

\title{
TCR deep sequencing of transgenic RAG-1-deficient mice reveals endogenous TCR recombination: a cause for caution
}

\author{
Helen M McGuire ${ }^{1,2,3}$ Thomas S Watkins ${ }^{4,5,6}$ Matthew Field ${ }^{4}$, Sarah Taylor ${ }^{7}$ \\ Nao Yasuyama ${ }^{7}$ Andrew Farmer ${ }^{7}$ John J Miles ${ }^{4,5,6,8}$ Barbara Fazekas de St. Groth ${ }^{2,3}$ \\ 1. Centenary Institute, Camperdown, NSW, Australia \\ 2. Discipline of Pathology, School of Medical Sciences, Charles Perkins Centre, University of Sydney, NSW, Australia \\ 3. Ramaciotti Facility for Human Systems Biology, Charles Perkins Centre, University of Sydney, NSW, Australia \\ 4. Centre for Biodiscovery and Molecular Development of Therapeutics, AITHM, James Cook University, Cairns, QLD, \\ Australia \\ 5. QIMR Berghofer Medical Research Institute, Brisbane, QLD, Australia \\ 6. Faculty of Medicine, The University of Queensland, Brisbane, QLD, Australia \\ 7. Takara Bio USA, Inc., CA, USA \\ 8. Institute of Infection and Immunity, Cardiff University School of Medicine, Cardiff, UK
}

\section{ABSTRACT}

The utility of T-cell receptor (TCR) transgenic mice in medical research has been considerable, with applications ranging from basic biology all the way to translational and clinical investigations. Crossing of TCR transgenic mice with either recombination-activating gene (RAG)-1 or RAG-2 knockouts is frequently used to generate mice with a monoclonal T-cell repertoire. However, low level productive TCR rearrangement has been reported in RAGdeficient mice expressing transgenic TCRs. Using deep sequencing, we set out to directly examine and quantify the presence of these endogenous TCRs. Our demonstration that functional nontransgenic TCRs are present in nonmanipulated mice has wide reaching ramifications worthy of critical consideration.

We have used 5' RACE and deep sequencing to quantitatively measure productively rearranged endogenous T-cell receptor (TCR) transcripts in TCR transgenic recombinationactivating gene (RAG)-1-deficient mice. Our sequence data highlight an as yet unappreciated mechanism by which productively rearranged TCRs are generated in the absence of RAG-1 gene expression.

TCR transgenic mice are often utilized by researchers to investigate the function of antigenspecific T cells. A number of factors, including incomplete allelic exclusion of the TCR $\alpha$ chain, 
allow expression of a repertoire of nontransgenic, endogenously rearranged TCRs in such transgenic mice. ${ }^{1}$ Breeding TCR transgenic mice on to a background deficient in either RAG-1 or RAG-2 is frequently employed to generate mice with a monoclonal T-cell repertoire encoded by the transgenes. ${ }^{2}$ We have previously reported selection of a population of T cells expressing the transgene-encoded TCR $\beta$ chain but not the transgene-encoded TCR $\alpha$ chain in 5C.C7 TCR transgenic mice on a RAG-1-deficient background.3 Such cells were overrepresented within effector T cells in the colon of mice that spontaneously developed bowel inflammation, suggestive of T-cell proliferation driven by a nontransgenic TCR specificity. More recently, it has been reported that transfer of T cells from TCR transgenic RAG-2deficient mice into allogeneic hosts resulted in accumulation of donor-derived polyclonal T cells expressing endogenously rearranged, functional, nontransgenic TCR $\alpha$ and TCR $\beta$ chains, once again supporting the existence of functional, nontransgenic TCRs within the T-cell repertoire of TCR transgenic RAG-deficient mice. ${ }^{4}$ While the latter study was informative, the sequencing of TCR transcripts was limited in depth to indirect measurement of expanded T cells recovered from allogeneic hosts, using low-resolution immunoscope studies. Strong selective pressure has also been reported to drive TCR revision, in which transgene-encoded TCR $\beta$ chains are replaced by endogenous rearrangements. This phenomenon requires peripheral re-expression of RAG-1 and RAG-2, and is absent in RAG-deficient mice. ${ }^{5}$

Here, we have utilized the new 5'-RACE-based SMARTer Mouse TCR $\alpha / \beta$ Profiling Kit (Takara Bio USA, Inc.), to perform in-depth sequencing of TCRs in five organs in each of three unmanipulated 5C.C7 transgenic RAG-1/- mice with no evidence of inflammation or autoimmunity. The mice varied in age from 3 to 9 months. Paired-end 300 base pair reads were obtained by Illumina sequencing and aligned and assembled using MiXCR. ${ }^{6}$ Across all samples, we determined 12725331 reads for TCR $\alpha$ and 4104331 reads for TCR $\beta$. TCRs that did not correspond to the transgenic TCR $\alpha$ and TCR $\beta$ chains were manually checked using NCBI Megablast to ensure that they contained at least $300 \mathrm{bp}$ of somatic $V$ gene sequence $3^{\prime}$ of complementarity-determining region 3 (CDR3), plus the entire somatic J gene sequence $5^{\prime}$ of CDR3. This excluded a large number of sterile (unrearranged) TCR $\alpha$ transcripts identified by miXCR, which comprised between 1 and 3\% of all TCRa sequences, with the highest representation in the thymic samples. 
Consistent with our previous flow cytometric analysis in 5C.C7 transgenic RAG-1 ${ }^{-/-}$mice, ${ }^{3}$ we did not identify any functional TCR $\beta$ chain reads resulting from endogenous $V(D) J$ recombination. We identified eight unique TCR $\alpha$ rearrangements using four distinct T cell receptor alpha variable (TRAV) genes and seven T cell receptor alpha joining (TRAJ) regions within the 15 samples (Figure 1). Six of the eight rearrangements contained $\mathrm{N}$ region nucleotides and all appeared to result from RAG-dependent cleavage at the appropriate intron-exon boundaries of the TRAV and TRAJ genes.

A distinct bias toward use of proximal TCR $\alpha$ gene segments was seen, with the four TRAV genes clustered within $300 \mathrm{~kb}$ of the $3^{\prime}$-end of the $1800 \mathrm{~kb}$ TRAV gene locus. Of the 18 TRAV genes in this $300 \mathrm{~kb}$ interval, only three also function as TRDV genes, but all three were rearranged in the endogenous TCR $\alpha$ chains we identified. The marked preference toward proximal TRAV genes and particularly TRAV/T cell receptor alpha diversity genes is suggestive of rearrangement at a relatively early stage of thymic differentiation. ${ }^{7}$ TRAJ usage was also biased toward proximal genes, with six of the seven TRAJ regions within the $5^{\prime}$ half of the TRAJ locus.

The distribution of endogenously rearranged TCR $\alpha$ chains within different organs from each animal indicated that rearrangements of TRAV21/DV12 to TRAJ43 and TRAJ45 were present in each of the three animals, while functional transcripts containing TRAV16 and TRAJ56 were shared by two of the three (Figure 2). These two TRAV regions comprised only a minor fraction of the peripheral repertoire of control C57BL/6 mice (less than 5\% and 1\%, respectively, data not shown), once again consistent with nonrandom use of TRAV and TRAJ segments in the TCR transgenic RAG-deficient mice.

The cellular distribution of endogenous TCR $\alpha$ chain transcripts within each animal was consistent with postrearrangement selection of functional TCRs in response to antigen, with increased representation of individual rearrangements in the periphery compared with the thymus. In each animal, one to two individual clones were present in the large intestine and were also dominantly represented in all peripheral lymphoid tissues sampled. The oldest mouse had the highest number of functional endogenously rearranged transcripts, including two clones that together accounted for $1 \%$ of transcripts within the large intestine, while 
$0.67 \%$ of intestinal transcripts in the youngest mouse were encoded by a nontransgenic, endogenously rearranged TCR $\alpha$.

In conclusion, we found functional TCR recombination events in 5C.C7 transgenic RAG$1^{-/-}$mice, suggesting only a single version of RAG (in this case RAG-2) is required for successful TCR $\alpha$ VJ recombination in mice. Such rare recombination events, which are not detected in nontransgenic RAG-1/- mice, may be preserved in cells where transgene-encoded expression of a functional TCR $\beta$ chain supports thymic development. Even in young animals kept under SPF conditions, T cells with endogenous specificities are preferentially selected into the peripheral TCR repertoire and could introduce highly biologically relevant and unintended outcomes. In summary, caution is advised when interpreting data from TCR transgenic mice. 


\section{REFERENCES}

1 Bluthman $\mathrm{H}$, Kisielow $\mathrm{P}$, Uematsu $\mathrm{Y}$, et al. T-cell-specific deletion of T-cell receptor transgenes allows functional rearrangement of endogenous $a-$ and $b-$ genes. Nature 1988; 334: 156-159.

2 Shinkai Y, Koyasu S, Nakayama K-I, et al. Restoration of T cell development in RAG-2deficient mice by functional TCR transgenes. Science 1993; 259: 822-825.

3 Koh W-P, Chan E, Scott K, et al. TCR-mediated involvement of $\mathrm{CD}^{+}$transgenic T cells in spontaneous inflammatory bowel disease in lymphopenic mice. J Immunol 1999; 162: 72087216.

4 Montaudouin C, Boucontet L, Mailhe-Lembezat MP, et al. Endogenous TCR recombination in TCR Tg single RAG-deficient mice uncovered by robust in vivo T cell activation and selection. PLoS One 2010; 5: e10238.

5 Hale JS, Ames KT, Boursalian TE, Fink PJ. Cutting Edge: rag deletion in peripheral T cells blocks TCR revision. J Immunol 2010; 184: 5964-5968.

6 Bolotin DA, Poslavsky S, Mitrophanov I, et al. MiXCR: software for comprehensive adaptive immunity profiling. Nat Methods 2015; 12: 380-381.

$7 \quad$ Thuderoz F, Simonet MA, Hansen O, et al. Numerical modelling of the $V-J$ combinations of the T cell receptor TRA/TRD locus. PLoS Comput Biol 2010; 6: e1000682 


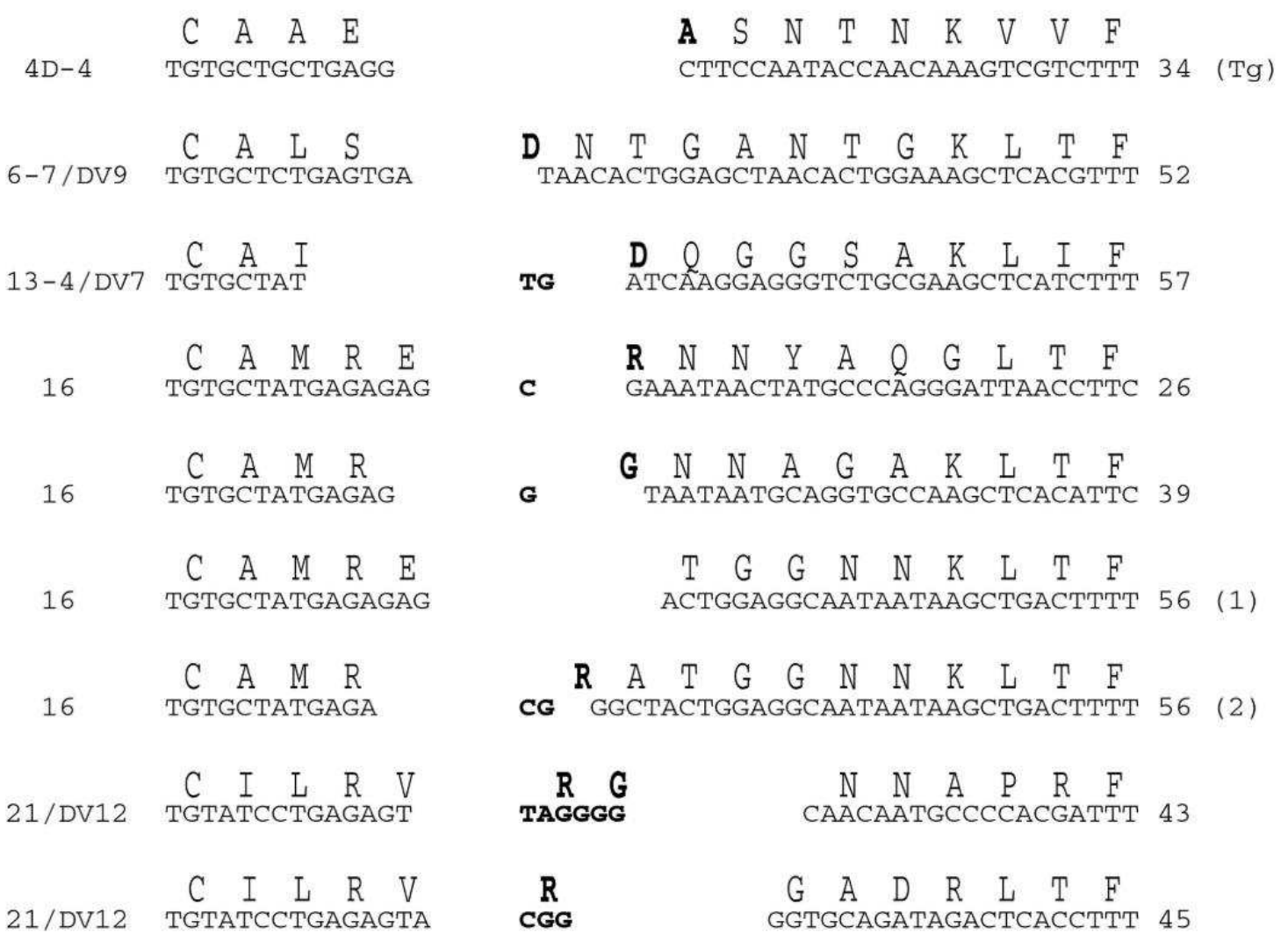

Endogenous TCR $\alpha$ recombination in 5C.C7 transgenic RAG-1/- mice. Junctional nucleotide and amino acid sequences from the TRAV4-TRAJ34 5C.C7 transgene and the eight endogenous TCR $\alpha$ rearrangements identified in 5C.C7 transgenic RAG-1/-- mice. Germline TRAV sequences are on the left, germline TRAJ sequences on the right and $\mathrm{N}$-region nucleotides in bold are at the center. Amino acids encoded by the V-J junction are in bold. Two distinct TRAV16-TRAJ56 rearrangements were identified, indicated as (1) and (2). 


\section{FIGURE 2}

TCR deep sequencing of transgenic RAG-1-deficient mice reveals endogenous TCR recombination: a cause for caution
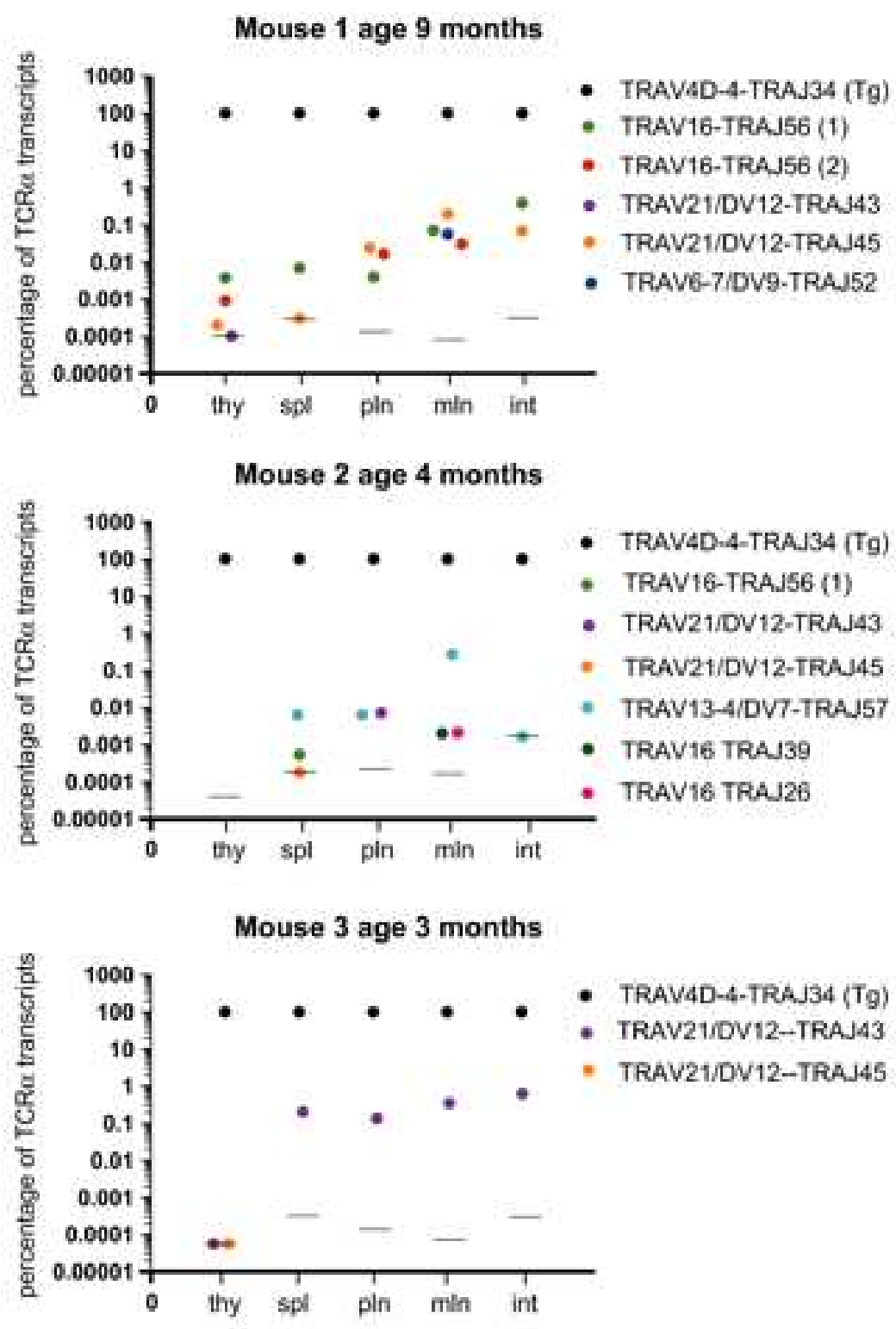

Frequency of endogenous TCR $\alpha$ transcripts in 5C.C7 transgenic RAG-1/- mice. Five organs from each of three mice (Mouse 1, age 9 months; Mouse 2, age 4 months; Mouse 3 age 3 months) were analyzed and the percentage of each functional TCR $\alpha$ transcript calculated. Transcripts coded with the same color had identical junctional nucleotide sequences. The bars represent the percentage corresponding to a single transcript in each organ. Thy, thymus; spl, spleen; pln, peripheral lymph nodes; $\mathrm{mln}$, mesenteric lymph nodes; int, large intestine. 